\title{
Identifying Potential Re-Entrant Circuit Locations From Atrial Fibre Maps
}

\author{
Max Falkenberg ${ }^{1,2,3, *}$, David Hickey ${ }^{1, *}$, Louie Terrill ${ }^{1, *}$, Alberto Ciacci ${ }^{1,2,3}$, \\ Nicholas S Peters ${ }^{3}$, Kim Christensen ${ }^{1,2,3}$ \\ ${ }^{1}$ Blackett Laboratory, Imperial College London, London, United Kingdom \\ ${ }^{2}$ Centre for Complexity Science, Imperial College London, London, United Kingdom \\ ${ }^{3}$ ElectroCardioMaths Programme, Imperial College London, London, United Kingdom \\ * These authors contributed equally to this paper
}

\begin{abstract}
Re-entrant circuits have been identified as potential drivers of atrial fibrillation (AF). In this paper, we develop a novel computational framework for finding the locations of re-entrant circuits from high resolution fibre orientation data. The technique follows a statistical approach whereby we generate continuous fibre tracts across the tissue and couple adjacent fibres stochastically if they are within a given distance of each other. By varying the connection distance, we identify which regions are most susceptible to forming re-entrant circuits if muscle fibres are uncoupled, through the action of fibrosis or otherwise. Our results highlight the sleeves of the pulmonary veins, the posterior left atrium and the left atrial appendage as the regions most susceptible to re-entrant circuit formation. This is consistent with known risk locations in clinical AF. If the model can be personalised for individual patients undergoing ablation, future versions may be able to suggest suitable ablation targets.
\end{abstract}

\section{Introduction}

Atrial fibrillation (AF) is the most common cardiac arrhythmia causing harmful complications such as stroke [1]. A full mechanistic understanding of AF is still lacking and, as a result, treatment options have disappointing success rates [2]. Transmural re-entrant circuits have been identified as a potential mechanism of AF capable of explaining both focal and re-entrant activity [3,4].

In this proof of concept paper, we introduce a novel computational model of the atria for identifying potential locations of re-entrant circuits. Continuous fibre tracts are generated from sheep heart fibre orientation data forming a realistic atrial topology [5].

Personalised heart models have been a growing focus in computational cardiology. Many existing models focus on correlating patterns of fibrosis in the myocardium to the emergence and maintenance of re-entrant drivers in AF [6-8]. Other models focus explicitly on muscle fibre orientation to assess the effect of fibre heterogeneity on atrial electrical propagation patterns and potential re-entry locations $[5,9,10]$. These models typically study the dynamics of spiral waves, which maintain $\mathrm{AF}$, using reactiondiffusion type equations where conduction velocities differ along fibres as opposed to across fibres. However, fibres are not explicitly decoupled - the tissue is a continuous substrate through which electrical signals can propagate.

In this paper, our aim is to identify the locations where decoupling neighbouring fibre tracts creates a substrate for micro-anatomic re-entries which can initiate fibrillation. Our approach is statistical - we generate many possible fibre maps from the known fibre orientation data, identify the potential locations where a re-entrant circuit may form, and collate the results to find locations which are consistently prone to the formation of re-entrant circuits. We do not directly investigate the behaviour of spiral waves since our focus is purely structural, avoiding the need to solve the reaction diffusion equations typically used in cardiac electrophysiology models. This allows the generation of very large statistics not accessible using other methods.

\section{Methods}

The algorithm for identifying re-entrant circuits is inspired by percolation based physics models where cardiomyocytes are coupled strongly along muscle fibres, but weakly across fibres [11-14]. Re-entrant circuits can be sustained if the path length of a circuit exceeds the refractory wavelength, calculated as the product of the effective refractory period and the conduction velocity. The parameters used to calculate these values can be found in [12]. Using the sheep heart data, we generate the principal fibres across the atria and couple adjacent fibres with a probability dependent on their proximity. Having generated the fibre maps, we identify closed loops of length longer than a predefined refractory wavelength as potential locations for 
re-entrant circuits.

\subsection{Constructing fibre maps}

A high-resolution $(50 \mu m \times 50 \mu m \times 50 \mu m$ voxels $)$ image-based map of atrial myofibre orientations for a sheep heart was acquired [5]. The map takes the form of a vector field, giving the predominant fibre directions relative to a fixed spatial co-ordinate system. For the sake of computational simplicity, we coarse-grained the vector field by taking the average fibre orientation within a cubic block of voxels. We use a coarse graining of 6 cells $(300 \mu \mathrm{m})$ to ensure both sufficiently high anatomical detail and the computational feasibility necessary for large statistics.

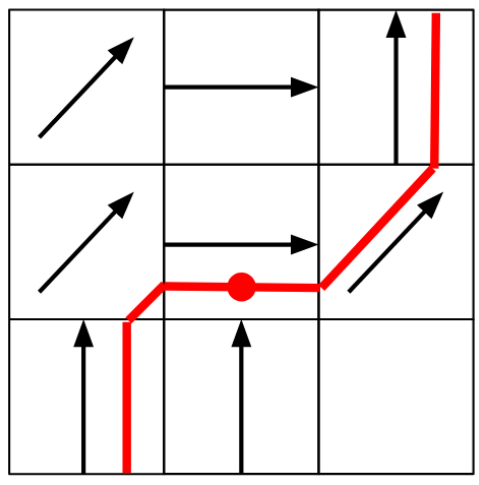

Figure 1. Illustration of the FACT algorithm on a simplified 2D grid. Each voxel has a linear scale of $300 \mu \mathrm{m}$ after coarse graining. The red dot indicates the starting position, and the red line is the fibre traced out by the algorithm.

Fibres were generated by adapting the Fibre Assignment by Continuous Tracking (FACT) algorithm, which performs linear interpolation in the direction of the fibre orientation within each voxel of the vector field, see Fig. 1 [15]. The algorithm is well-established in neuroscience for tracking neurons and is simple to implement [16].

Fibres were generated starting from the centre of every non-empty coarse-grained voxel. This introduced a significant overdensity of fibres and density fluctuations dependent on the orientation of fibres relative to the Cartesian co-ordinate system. Although there is a natural variation in fibre density across the atria, our dataset does not include this information.

The Spherical-deconvolution informed filtering of tractograms (SIFT) algorithm was adapted to correct for the overdensity of fibres and the co-ordinate system based density fluctuations [17]. SIFT was chosen for its relative speed compared to alternative algorithms and for the lack of assumptions required in its implementation.

The fibres generated using FACT and SIFT are not cou- pled. To couple fibres, nodes were seeded at regular intervals of approximately the voxel length along the fibres. Nodes in separate fibres were then coupled according to their proximity using a sigmoidal probability density function

$$
P_{\text {coupled }}(r)=\frac{1}{e^{s(r-c)}+1},
$$

where $r$ is the distance between nodes in units of the voxel spacing, $s=7$ is the steepness of the transition, and $c$ is a characteristic distance for the connection probability. This probability function is suitable since it is 1 for small distances and approaches zero quickly for $r>c$. Results are robust against changes in the steepness, $s$. A small region of an example fibre map can be seen in Fig. 2.

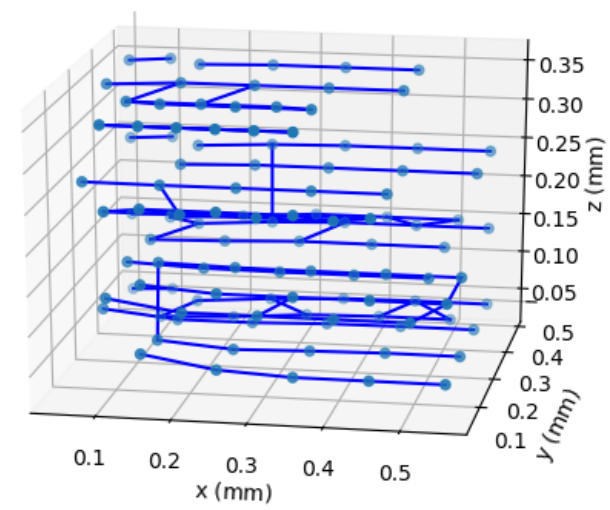

Figure 2. Example result of the fibre generation process. Points represent nodes; lines represent connections between nodes. Fibres principally lie along the $\mathrm{x}$-axis with fewer connections in the $\mathrm{y}$ - and $\mathrm{z}$ - directions.

The overall level of coupling is controlled by changing the characteristic distance. If $c$ is too small, fibres are completely isolated and do not form a closed loop. If $c$ is large, fibres are strongly coupled and the closed loops formed are shorter than the refractory wavelength required by a re-entrant circuit. In a transition region fibres are coupled sufficiently to allow conduction across the tissue, but the closed loops that form have path length greater than the refractory wavelength. These regions are identified as potential locations for re-entrant circuits.

From the fibre map, circuits are identified by generating signals at the sinus node and letting the signal diffuse across the network. By simulating stochastic unidirectional conduction block, we identify locations where the signal spreading from the sinus node forms a continuously excited circuit with path length greater than the refractory wavelength, see [13] for details.

The methods used here are probabilistic. No single fibre map generated is an exact representation of the muscle fibre coupling observed in a real heart. However, by averag- 
(a)

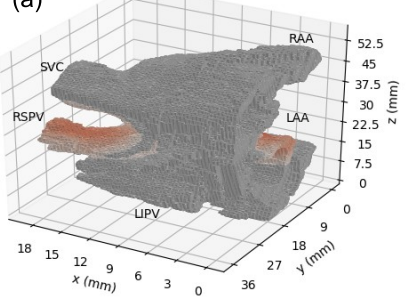

(b)

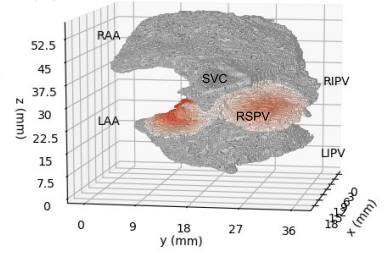

(c)

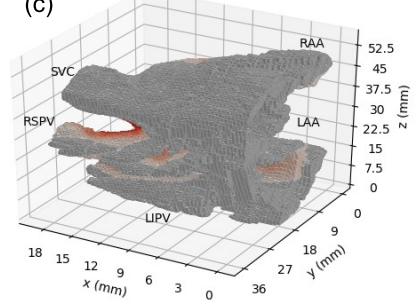

(d)

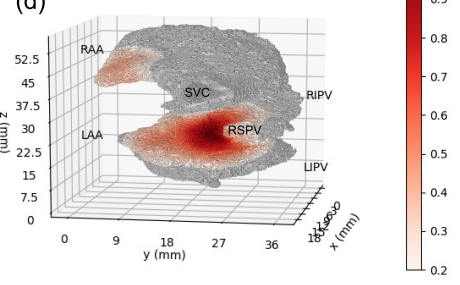

Figure 3. Risk probability of re-entrant circuits across the atria for moderately decoupled tissue (a \& b), and significantly decoupled tissue (c \& d), for two different views of the same model. The superior vena cava (SVC), right/left superior/inferior pulmonary veins (RSPV/LSPV/RIPV/LIPV) and the right/left atrial appendages (RAA/LAA) are indicated. For moderately decoupled tissue, absolute risk is low - key risk regions correspond to the posterior left atrium (PLA, region highlighted between RIPV and LIPV), RSPV and LAA. As tissue is decoupled further, the risk region covers a significant fraction of the atrial tissue, specifically the left atrium and the RAA. A spherical convolution has been used for visual clarity. Risk regions with probability less than 0.25 are shown in grey for contrast.

ing over many realisations, we can identify regions which are consistently more at risk of decoupling and forming re-entrant circuits.

\section{Results}

To assess whether the fibre maps generated are reasonable, we compare the activation times for the networks generated using our algorithm to a randomised fibre map with isotropic connection probabilities (figure not shown). The results are consistent with previous studies using the sheep heart fibre map where the propagation of signals from the sino-atrial node to the left atrium, simulated using the Fenton-Karma model, was faster using the real fibre data than randomised isotropic data [5].

To identify locations in the atria susceptible to the formation of re-entrant circuits, we generate 1000 randomised instances of the fibre map from the fibre orientation data for each parameter set. For each instance, we apply the circuit location algorithm and note the positions of any circuits identified. We then collate the data from all 1000 realisations and plot a heatmap of the probability that a given region in the atria is susceptible to the formation of re-entrant circuits, see Fig. 3. This result is sensitive to the choice of characteristic distance in Eq. (1). A characteristic distance of $c=1$ corresponds to 1 voxel spacing in the fibre map. For $c>1.2$, no re-entrant circuits are found. As $c$ is reduced, the left atrial appendage (LAA), the posterior left atrium (PLA) and the pulmonary veins (PVs), most notably the right superior PV, are identified as the primary risk locations for re-entrant circuits. If $c$ is reduced further, the risk locations cover a large fraction of the atrial surface, notably the left atrium and the RAA. The reduction in the characteristic distance can be thought of as decoupling neighbouring muscle fibres through the action of diffuse, interstitial fibrosis, that accumulates with age, or otherwise.

\section{Discussion}

This proof of concept work demonstrates that the regions believed to play critical role in the emergence and maintenance of focal drivers in AF (PVs, LAA and PLA) can be identified as risk regions for the formation of reentrant circuits from fibre orientation data only. We do not consider the emergence of focal drivers from cardiomyocyte automaticity. Interestingly, the framework suggests that the first regions which harbor re-entrant circuits correlate to those targeted using various ablation strategies which have success for AF [2]. However, as fibres decouple further, the risk region covers a significant fraction of the atrial tissue such that the electrical isolation of any one region through ablation is unlikely to prevent fibrillation. Equally, the risk region is so vast, especially in the left atrium, that too many individual sources may exist for a focal ablation strategy to be successful. This may partially explain why persistent $\mathrm{AF}$ is in some cases untreatable using current ablation strategies. The model also shows a greater density of risk regions in the left atrium which is consistent with clinical observations that $70 \%$ of all focal triggers in persistent AF are found in the left atrium [18].

\subsection{Limitations of study \& Future work}

The work presented here is early stage and should be treated as a proof of concept. The results are derived from a single sheep atria fibre map, and as such the results may not be representative of other hearts with unique fibre orientations, or of human AF. Testing the methods on a range of other fibre maps and validating the risk regions clini- 
cally should be a key focus moving forward. Work is ongoing applying our methods to a human bi-atrial fibre map acquired in [19].

A major limitation in the current work is the small voxel size used to generate the fibre map. After coarse graining, each voxel has a linear dimension of approximately $300 \mu \mathrm{m}$. This is significantly smaller than the smallest voxel size achievable in a cardiac MRI. Non-invasive methods are unlikely to be able to generate fibre maps with the resolution necessary for these techniques to have clinical benefit. Adapting our model to work at lower fibre orientation resolutions may bring us closer to a framework which has predictive power relevant clinically.

Our approach assumes uniform decoupling of muscle fibres throughout the atria, simulating the accumulation of diffuse, interstitial fibrosis. However, patchy and compact fibrosis are also known to aid the formation of re-entrant circuits. To generate personalised models for individual hearts, the method for generating fibre connections must take account of realistic fibrosis patterns.

\section{Conclusion}

We have introduced a framework for identifying potential locations of re-entrant circuits from atrial fibre maps. The technique successfully identifies the importance of the PVs, PLA, and the LAA to AF, but also indicates that if muscle fibres decouple sufficiently, re-entrant circuits can form across a large portion of the atrial tissue, complicating prospective ablation strategies.

\section{Acknowledgements}

MF is grateful to Jichao Zhao for providing the sheep heart fibre orientation data. MF and AC acknowledge PhD studentships from the UK EPSRC. NSP acknowledges funding from the British Heart Foundation (RG/16/3/32175 and Centre of Research Excellence), the Rosetrees Trust, and the National Institute for Health Research (UK) Biomedical Research Centre.

\section{References}

[1] Benjamin EJ, et al. Impact of atrial fibrillation on the risk of death: the framingham heart study. Circulation 1998; 98(10):946-952.

[2] Calkins H, et al. 2017 HRS/EHRA/ECAS/APHRS/ SOLAECE expert consensus statement on catheter and surgical ablation of atrial fibrillation. Heart Rhythm 2017; 14(10):e275-e444.

[3] Hansen BJ, et al. Atrial fibrillation driven by microanatomic intramural re-entry revealed by simultaneous subepicardial and sub-endocardial optical mapping in explanted human hearts. European Heart Journal 2015; 36(35):2390-2401.
[4] Nattel S, et al. Demystifying rotors and their place in clinical translation of atrial fibrillation mechanisms. Nature Reviews Cardiology 2017;14(9):509-520. ISSN 1759-5002.

[5] Zhao J, et al. An image-based model of atrial muscular architecture: effects of structural anisotropy on electrical activation. Circulation Arrhythmia and Electrophysiology 2012;5(2):361-370.

[6] Roney $\mathrm{CH}$, et al. Modelling methodology of atrial fibrosis affects rotor dynamics and electrograms. EP Europace 12 2016;18:iv146-iv155.

[7] Vigmond E, et al. Percolation as a mechanism to explain atrial fractionated electrograms and reentry in a fibrosis model based on imaging data. Heart Rhythm 2016; 13(7):1536 - 1543. ISSN 1547-5271.

[8] Schwarz EL, et al. Patient-derived models link re-entrant driver localization in atrial fibrillation to fibrosis spatial pattern. Cardiovascular Research 04 2016;110(3):443-454. ISSN 0008-6363.

[9] Tobón C, et al. A three-dimensional human atrial model with fiber orientation. electrograms and arrhythmic activation patterns relationship. PLOS ONE 02 2013;8(2):1-13.

[10] Ramlugun GS, et al. Dynamics of cardiac re-entry in microCT and serial histological sections based models of mammalian hearts. ArXiv eprints Sep 2018;arXiv:1809.01186.

[11] Moe G, et al. A computer model of atrial fibrillation. American Heart Journal 1964;67(2):200 - 220. ISSN 0002-8703.

[12] Christensen K, et al. Simple model for identifying critical regions in atrial fibrillation. Physical Review Letters 2015; 114(2):028104.

[13] Falkenberg M, et al. Unified Mechanism of Atrial Fibrillation in a Simple Model. ArXiv eprints Oct 2018; arXiv: 1810.12062 .

[14] McGillivray MF, et al. Machine learning methods for locating re-entrant drivers from electrograms in a model of atrial fibrillation. Royal Society Open Science 2018;5(4):172434.

[15] Mori S, et al. Three-dimensional tracking of axonal projections in the brain by magnetic resonance imaging. Annals of Neurology 1999;45(2):265-269.

[16] Jiang H, et al. Dtistudio: resource program for diffusion tensor computation and fiber bundle tracking. Computer Methods and Programs in Biomedicine 2006;81(2):106116.

[17] Smith RE, et al. Sift: spherical-deconvolution informed filtering of tractograms. Neuroimage 2013;67:298-312.

[18] Haissaguerre M, et al. Driver domains in persistent atrial fibrillation. Circulation 2014;130(7):530-538.

[19] Wang Y, et al. A robust computational framework for estimating $3 \mathrm{~d}$ bi-atrial chamber wall thickness. Computers in Biology and Medicine 2019;114:103444. ISSN 0010-4825.

Address for correspondence:

Max Falkenberg

Centre for Complexity Science, Imperial College London, London SW7 2AZ, United Kingdom

max.falkenberg13@imperial.ac.uk 